\title{
La colonisation de quelques grottes du Jura par Niphadobota alpina Bezzi (Dipt. Tipulidae)
}

par

\section{M.-J. TURQUIN*}

Lors de l'étude biocoenotique du Puits de Rappe (Turquin, 1971) j'ai capturé 6 individus ( 4 femelles et 2 mâles) du Diptère Tipulidé aptère Chionea (s. lato). Ces insectes ont depuis longtemps intrigué les biologistes européens et américains; de nombreuses références sont citées par Bitsch (1955) qui fait le point des stations françaises à l'occasion de la capture de Chionea (=Niphadobota) lutescens le 31-1-1955, dans une souche d'arbre, sous la neige, dans la Côte d'Or. C'était environ le 14ème exemplaire du genre signalé en France. Les stations citées dans la littérature sont les suivantes:

1. Meurthe et Moselle (à Buré) 6 ind. dans une tanière de Blaireau, capturés par Heim de Balsac (1934), N. lutescens.

2. Vosges (Lac Blanc): ? (in Heim de Balsac, 1934).

3. Haut-Rhin (près de Mulhouse) 2 ind. (Heim de Balsac, 1934).

4. Jura (?) en 1821. Bezzi suppose qu'il s'agit de $N$. lutescens (in Heim de Balsac, 1934).

5. Basses-Alpes ("grotte" de Cousson à $1500 \mathrm{~m}$ d'altitude) 1 ind. en 1906 et 1 en 1911 par Peyerimhoff (1906) qui le détermine comme Chionea araneoïdes. Bezzi (in Heim de Balsac, 1934) pense qu'il s'agit peut-être de Chionea (=Niphadobota) alpina, étant donné l'altitude de la capture; Pierre (1924) cite $N$. alpina à Cousson et dans les Alpes.

6. Puy de Dôme (Puy de Clierzou) 2 ind. Teilhard, Du Buysson et Villeneuve (in Pierre, 1924) N. lutescens.

7. Haute-Loire (?) N.alpina (in Bitsch, 1955)

8. Côte d'or (Sombernon) 1 femelle, Bitsch (1955) N. lutescens.

En 1969, Burghele-Balacesco procède à une révision du genre Chionea; se basant sur les travaux d'Enderlein (sur le nombre d'articles antennaires) et de Kratochvil (sur la structure de l'appareil génital mâle), elle écrit: "les différences constatées dans la structure de l'organe copulateur mâle entre les deux groupes d'espèces (longicornes et brevicornes) sont si grandes que leur division en deux genres distincts s'impose, ce qu'à déjà fait Enderlein en 1936 d'après le nombre des articles antennaires. Nous soutenons donc pleinement la proposition d'Enderlein de séparer ce groupe en deux genres: Chionea Dalman et Niphadobota Enderlein". L'auteur

* Collaboration technique de Mme. E. Michalon. Laboratoire de Biologie souterraine. Dept. Biologie Animale at Zoologie - Université Claude Bernard Lyon 1 - 43 Boulevard du 11 novembre -69100 - Villeurbanne. 


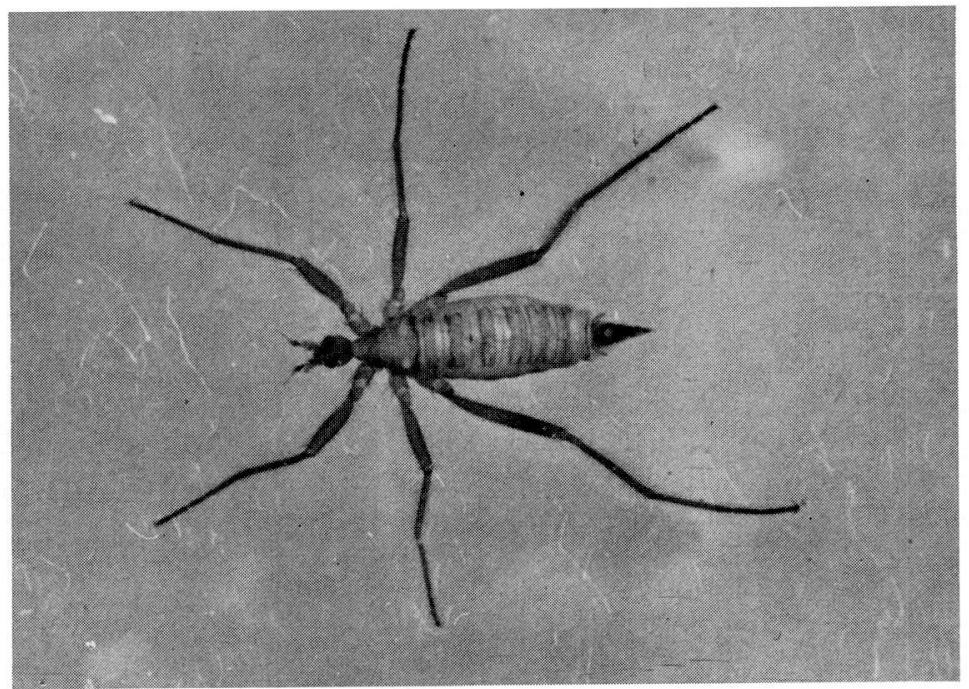

Niphadobota alpina (Diptère, Tipulidé): individu femelle (x 10)

décrit six espèces actuellement connues en Europe centrale et méridionale:

Niphadobota alpina Bezzi, 1908

N. lutescens Lundström, 1907

N. kratochvili Burghele-Balacesco, 1969

N. botoseanui Burghele-Balacesco, 1969

Chionea minuta Tahvonen, 1932

C. racovitzai Burghele-Balacesco, 1969

De plus, elle considère que l'espèce Chionea italica Venturi, 1956 est synonyme de $N$. alpina pour les mâles, de $N$. lutescens pour la femelle.

Le genre Chionea disparait donc de la faune française au profit de Niphadobota.

Grâce à la révision de Burghele-Balacesco il est désormais aisé de différencier les espèces alpina et lutescens surtout pour les individus mâles et il serait intéressant de retrouver les stations de Cousson et du Puy de Clierzou pour déterminer ces Diptères, avec les nouveaux critères définis en 1969.

A la suite de la capture de $N$. alpina au puits de Rappe (Ain) j'ai piégé durant les hivers $70-71$ et $71-72$ la zone d'entrée de diverses grottes du Jura méridional depuis Montfleur (Jura) au Nord jusqu'à Hauteville (Ain) au Sud. Deux stations nouvelles seulement ont été trouvées: 1 individu femelle à la grotte de Challes (Ain) et une femelle à la grotte laboratoire de Hautecourt (Ain) (Turquin, 1972); le tableau résume les conditions de milieu de ces trois stations. Cette rareté des captures est conforme à la biogéographie et à l'écologie du genre Chionea s. lato.

En effet, ces Tipulidés, "snowflies" des Anglo-saxons, sont données par M.S. Mani (1962) comme caractéristiques des Diptères de la faune nivale de l'Himalaya, 
où ils vivent à peu de distances des névés, dans la zone humide. Heim de Balsac (1934) écrit: "les Chionea sont des éléments très représentatifs de la faune des régions froides de l'Eurasie et de l'Amérique du Nord. Dans cette vaste région holarctique, le nombre des espèces et des individus décroit du Nord au Sud ... Sous des latitudes moins élevées, dans l'Europe moyenne par exemple, le recensement des lieux de capture montre d'une part la dislocation de l'aire de répartition (qui se réduit à des zories de climat froid ou continental), d'autre part, la localisation résiduelle sur des massifs montagneux à mesure que la latitude s'abaisse". Niphadobota alpina, capturé plusieurs fois aux environs de $3000 \mathrm{~m}$ d'altitude dans des fissures de rocher (par Strinati, 1967, par exemple) est bien un insecte hypsobionte; tous les auteurs s'accordent à le déclarer cryobionte, puisque sa température optimale est au voisinage de $0^{\circ} \mathrm{C}$; dans l'Himalaya, sa période d'activité se situe entre $0^{\circ} \mathrm{C}$ et $-10^{\circ} \mathrm{C}$. Les auteurs canadiens signalent que les animaux pris à la main meurent très rapidement; ce sont, en effet, des sténothermes froids ou psychrophiles. Enfin, Krachtochvil (1936) relatant de nombreuses captures hivernales en Tchécoslovaquie, de $N$. lutescens sur la neige entre 180 et $1800 \mathrm{~m}$ d'altitude, conclut à l'habitat forestier de ces animaux, dont les larves seraient humicoles. Or, le Jura méridional en apparence loin de ces conditions de milieu extrêmes a été intéressé par les dernières glaciations quaternaires; l'influence de ces dernières sur la répartition des faunes cavernicoles (troglobies) aquatique et terrestre a déjà été étudiée plusieurs fois, en particulier par Ginet, (1953, 1972). Mais il s'agissait là surtout des conséquences catastrophiques de l'expansion des glaces sur un peuplement âgé, antérieur à l'évènement.

Les fossiles de grands Mammifères trouvés au Puits de Rappe nous permettent de connaître l'évolution des paysages depuis la fin du pléistocène. En effet, C. Guérin (communication personnelle) a reconnu des ossements de Bos primigenius, Cervus elaphus et Mammuthus primigenius qu'il rattache à la fin du Würm et associe à un biotope de forêt ouvert en climat relativement froid. Ce paléoclimat se réchauffe progressivement jusqu'au climat actuel. A une période très récente (à partir du XIIIème siècle) la déforestation conduit à l'assèchement des biotopes et à leur réchauffement (Aubert, 1969; Magnin, 1886). Le climat local devient beaucoup moins stable que sous un couvert forestier, plus chaud en été, plus froid en hiver, et moins humide. Tout concourt à restreindre de plus en plus l'habitat de Niphadobota alpina; ces animaux sont relégués dans les endroits les plus froids de la région: fonds de clolines ou entrées de grottes.

Le rôle de refuge exercé par les entrées de grotte pour une flore ou une faune rélicte a déjà été signalé par Vandel (1964), Gèze (1957), Condé (1955), Jeannel (1943). Citons le Chironomide à ailes réduites Cataliptus peyerimhoffi, qui vit dans les "tesserefts" algériens accompagné d'une florule arctique; le Diploure Plusiocampa caprai trouvé à l'état épigé dans les Alpes à $2300 \mathrm{~m}$ puis dans une grotte de Lombardie et dans une mine autrichienne; les Coléoptères des monts Bihar, en Transylvanie, troglobies vers $500 \mathrm{~m}$ d'altitude et qui "se retrouvent sous les pierres enfoncées à 100() $\mathrm{m}$, à la surface du sol vers 1200 à $1400 \mathrm{~m}$. Le Trichoptère Apatania zonella que j'ai capturé au Spitzberg et que Gislen et Brinck (1950) ont 


\section{Grotte de:}

\section{Situation \\ géographique}

Description

\section{Environnement}

\section{Substrat de}

la station

\section{Individus capturés}

Période de

piégeage

Températures au niveau des pièges

Humidité

\section{HAUTECOURT}

$\mathrm{X}=837,39 ; \mathrm{Y}=135,22$;

$$
\text { alt. }=464 \mathrm{~m}
$$

porche descendant, orienté à l'ouest.

Lande xérophile: taillis à Prunus mahaleb et Buxus sempervirens;

Juniperus communis

Talus d'éboulis avec débris végétaux abondants

$$
1 q
$$

fin février 1971

$$
<0^{\circ} \mathrm{C}
$$

présence de glace

\section{RAPPE}

$\mathrm{X}=834,44 ; \mathrm{Y}=127,44$

$$
\text { alt. }=300 \mathrm{~m}
$$

puits de $10 \mathrm{~m}$ au fond d'une doline ombragée

Prairies pâturées bouquet de Fagus silvaticus au fond de la doline.

Eboulis et talus de terre.

$$
4 \% ; 2 \text { o }
$$

Janvier 1971

$$
<0^{\circ} \mathrm{C}
$$

sol gelé

\section{CHALLES}

$\mathrm{Y}=842,45 ; \mathrm{Y}=129,45$; alt. $=600 \mathrm{~m}$

étroite galerie horizontale s'ouvrant á l'ouest dans un bois.

Forêt de Fagus silvaticus avec strate sous-frutescente de Buxus sempervirens.

Remplissage argileux sous un plancher stalegmitique.

19

début novembre 1971

$$
\cong 9^{\circ} \mathrm{C}
$$

atmosphère saturée 
découvert dans une grotte de l'ile de Gotland (Suède méridionale). Des exemples similaires sont exposés par Barr (1968) pour le continent américain.

Nous allons tenter de dégager d'après les exigences de ces insectes hypsobiontes, les composantes communes du milieu boréoalpin et du milieu hypogé, propres à les satisfaire. La liste des grandes tendances de spécialisations des insectes vivant dans la zone nivale, dressée par Mani $(1962,1968)$ va nous apporter les éléments de la réponse: aptérisme, réduction de la taille, sténothermie froide, hygrophilie, géophilie, régime détritivore. Tous ces caractères semblent correspondre aussi au régime de vie troglobie. J'après ce qui précède, les trois stations-refuge doivent présenter des caractéristiques spéciales les distinguant du milieu environnant, favorables à l'accomplissement du cycle vital du Diptère. Heim de Balsac, après les captures de Cousson et du puy de Clierzou conclut: "les faits... donnent à penser que les Chionea (Niphadobota) effectuent leur développement, au moins larvaire, dans le domaine souterrain".

Les relevés météorologiques effectués pendant 7 ans à la grotte de Hautecourt par R. Ginet et E. Michalon (voir aussi R. Ginet, 1970) permettent de définir de manière précise les variations microclimatiques annuelles d'un des biotopes hypogés de $N$. alpina. La configuration de la cavité (en "sac à air descendant", selon Gèze, 1957) fait que le porche représente un biotope singulièrement séparé de l'extérieur et même de l'intérieur de la grotte du point de vue thermique. Cette entrée de grotte constitue bien le "pôle du froid" de toute la région de Hautecourt, puisque sa moyenne annuelle est de $5,5^{\circ} \mathrm{C}$, contre $11,2^{\circ} \mathrm{C}$ à l'extérieur et $9,5^{\circ} \mathrm{C}$ au fond de la cavité (Fig. 1). En tenant compte des moyennes mensuelles (Fig. 2), on constate que le front froid se déplace plus avant dans le grotte de mai à octobre: sa température moyenne oscille de $0^{\circ} \mathrm{C}$, en janvier, à $7,5^{\circ} \mathrm{C}$, en septembre (mois le plus chaud dans la grotte). L'examen des relevés mensuels sur 7 ans montre que, pendant 6 mois, la température peut tomber en dessous de $0^{\circ} \mathrm{C}$ (de novembre à avril); bien que la moyenne annuelle soit basse, les variations de ce microclimat local sont néanmoins très amorties. A l'échelle macroclimatique, rappelons que l'isotherme $5^{\circ} \mathrm{C}$ est situé aux latitudes de Québec, des Iles Feroë, de Helsinki, de la Mongolie ou des Kouriles. En Europe, il faut s'élever au moins à 1300 m d'altitude pour subir une telle température.

Si on tient compte du fait que, dans le porche de Hautecourt, la moyenne mensuelle ne dépasse jamais $7,5^{\circ} \mathrm{C}$ en été, des conditions aussi rigoureuses ne se rencontrent que bien plus haut en montagne alpine (puisque la limite des forêts dans les Alpes coincide avec l'isotherme de juillet de $9,3^{\circ} \mathrm{C}$ ) et bien plus au Nord où l'influence de la latitude compense celle de l'altitude (cette frontière climatique des forêts nordiques suit à peu près l'isotherme $10^{\circ} \mathrm{C}$ du mois de juillet). Tout se passe comme si, climatiquement parlant, le porche de Hautecourt était situé au Nord de la Laponie, au nord du Kamtschatka ou au Groenland central!

Quant à la station du Puits de Rappe, à l'altitude de $300 \mathrm{~m}$, c'est aussi une grotte descendante, donc un piège à air froid: si dans les parties profondes, la température est supérieure à $12^{\circ} \mathrm{C}$, celle de l'entrée est très froide en hiver (aux alentours de $0^{\circ} \mathrm{C}$ de décembre à mars), en été, elle oscille autour de $9,5^{\circ} \mathrm{C}$.

La station la plus "chaude" en été $\left(9^{\circ} \mathrm{C}\right)$ est la petite grotte de Challes, à $600 \mathrm{~m}$ 


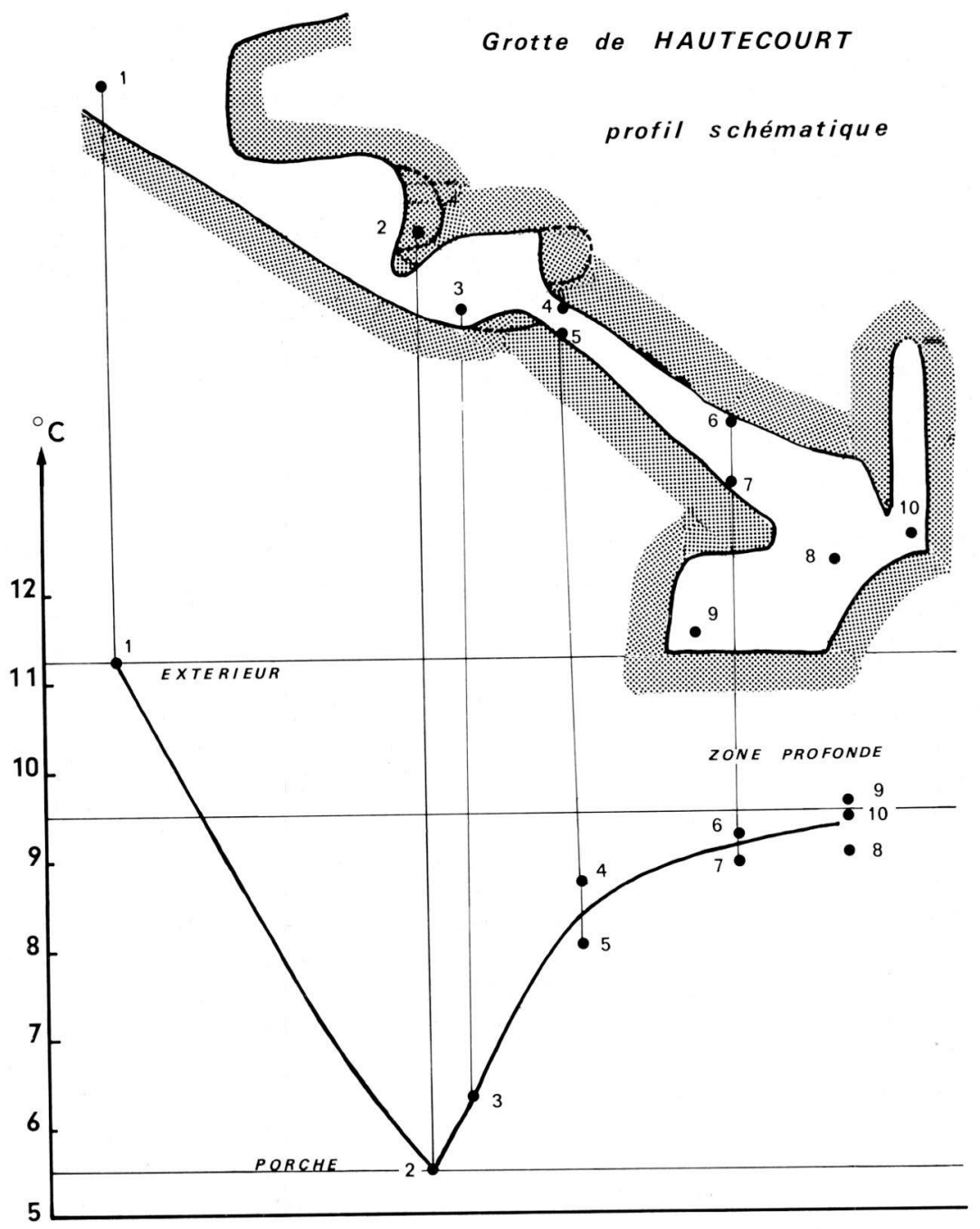

Fig. 1. Moyennes annuelles de températures observées à la grotte de Hautecourt en fonction de la profondeur. Documents R. Ginet, non publiés. (Point 3 = condensomètre, cf Ginet, 1970)

d'altitude. Ancienne exsurgence, ainsi qu'en témoigne un thalweg boisé, elle est constituée d'une galerie étroite et concrétionnée, sèche dans sa première partie horizontale. Une femelle de Niphadobota alpina a été trouvé au début de la partie humide, sous un plancher stalagmitique, au début novembre. C'est la capture la plus éloignée de l'entrée, et la plus précoce dans l'hiver. 


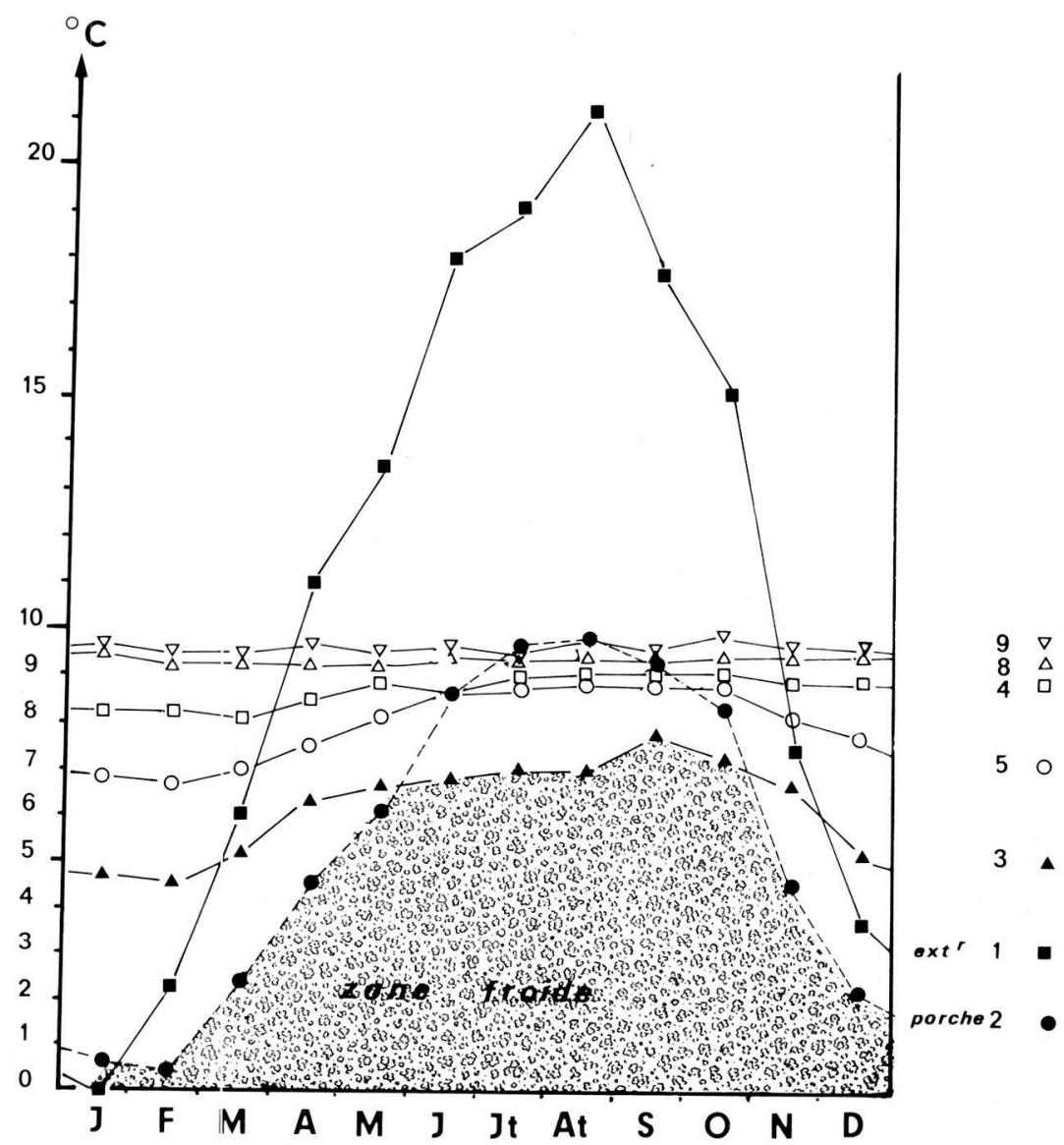

Fig. 2. Moyennes mensuelles de températures observées à la grotte de Hautecourt: les emplacements des mesures correspondent à la figure 1. En été le front froid se déplace vers le point 3. Documents R. Ginet.

Du cycle biologique de ces animaux, on ne connait, malheureusement, que la phase hivernale d'activité des adultes. Selon Heim de Balsac (1934), Egger et Frauenfeld en 1854 élevèrent "pendant quelque temps une larve de Chioneo (Niphadobota) lutescens"'(!). En montagne, les Diptères ont en général une seule génération, avec hibernation soit sous forme de larve, soit sous forme de pupe; l'adulte émerge en mai, et pond très rapidement, pour que le stade hivernant soit atteint. En effet, le facteur limitant est alors, non seulement la température, mais aussi l'absence d'eau libre, nécessaire à ces larves hygrophiles. Le cas de Niphadobota troglophile est exactement l'inverse de celui des Tipulides hypsobiontes: les adultes apparaissent de septembre à mars, avec un maximum en décembre et janvier; ils 
sont actifs pendant la période la plus froide de l'année, comme si le stade adulte était le plus psychrophile. Nous savons par ailleurs que la sténothermie subit des fluctuations calquées sur les conditions ambiantes pendant le cycle biologique d'un animal. Des élevages seraient intéressants à entreprendre, mais la rareté du matériel et sa fragilité font redouter une telle perspective.

Ainsi les trois nouvelles stations de $N$. alpina prouvent que l'espèce peut survivre en dessous de $1500 \mathrm{~m}$ d'altitude contrairement à l'opinion de Bezzi (in Heim de Balsac, 1934).

La physiologie de cet animal fait qu'à basse altitude il est strictement inféodé aux grottes froides. La rareté des stations semble démontrer que la dispersion des adultes théoriquement possible en hiver est promise à l'échec.

L'isolement de ces Tipulidés aptères est un nouvel exemple du mode de colonisation du milieu hypogé. Il est bien connu que les grottes en zone tempérée ont servi de refuge pour les espèces cryophiles, ancêtres des troglobies, au fur et à messure du réchauffement post-würmien. En effet, selon Barr (1968), la disparition de la faune cryophile du pleistocène n'a pas été soudaine: au stade $\mathrm{A}$, les animaux réfugiés dans les grottes froides n'étaient pas isolés génétiquement, mais au contraire avaient la possibilité de se croiser avec des congénères de surface à la faveur des transgressions de l'espèce durant les périodes froides (alternant avec des périodes plus chaudes pendant une phase glaciaire). Ce n'est qu'au moment du réchauffement final, peut-être même au fur et à mesure de la disparition de la forêt, que les populations sont devenues indépendantes, sans apport génétique de la surface (gene-flow): c'est la phase B. Barr en tire les conséquences: "dans ces petites colonies l'homozygotie conduit à une sélection sévère et à une diminution de la variabilité génétique; à ce moment (phase C) la probabilité de survie doit être faible ... Si la colonie survit, néanmoins, l'épigénotype se réorganise complètement, adaptant les animaux de manière précise à l'environnement, la variabilité génétique augmente (phase D) jusqu'à un niveau E où l'espèce est devenue troglobie". Les populations actuelles troglophiles de Niphadobota, dans le Jura méridional, suivant cette perspective, en seraient à la phase $\mathrm{C}$ de leur évolution vers un état troglobie éventuel.

\section{RESUME}

Les trois nouvelles localisations de Niphadobota alpina découvertes en France (Jura méridional) permettent à l'auteur de définir les exigences climatiques de l'espèce dont dépend étroitement sa biogéographie. L'origine glaciaire de la colonisation des grottes par ce Diptère est évoquée.

\section{SUMMARY}

Three new localities of Niphadobota (= Chionea) alpina in the French southern Jura allow the author to state that this insect's climatic requirements explain the biogeography of the species; the origin of the colonization of caves by this dipteran is considered. 


\section{BIBLIOGRAPHIE}

AUBERT, D. - 1969 - Phénomènes et formes du Kart jurassien. Eclogae geol. Helv. 62/2, $325-399$.

BARR, Th.C. Jr. - 1968 - Cave ecology and the evolution of troglobites. in Evolutionary Biology 2. DOBZHANSKY, Amsterdam.

BITSCH, J. - 1955 - Le genre Chionea (Diptera, Tipulidae). Ecologie, Systématique et morphologie. Trav. Lab. Zool. Stat. Aquicole Grimaldi, Fac. Sci. Dijon, 11, 1-26.

BURGHELE-BALACESCO, A. - 1969 - Révision des genres Chionea et Niphadobota en Europe centrale et méridionale (Dipt. Tipulidae). Ann. Soc. ent. France (N.S.), 5 (4), 983-1000.

CONDE, B. - 1955 - Matériaux pour une monographie des Diploures Campodéidés. Mém. Mus. Nat. Hist. Nat. Série A, Zoologie, XII, 1-202.

GEZE, B. - 1957 - La spéléologie scientifique. coll. "Le Rayon de la Science". Paris, Le Seuil.

GINET, R. - 1953 - Contribution à la connaissance de la faune cavernicole du Jura méridional. Influence des glaciations quaternaires. C.R. $1^{\circ}$ Cong. Intern. Spéléo. Paris, III, $125-1.30$.

GINET, R. - 1965 - Une réserve biologique souterraine: la grotte de Hautecourt. Spelunca Bull., 5 (1), 6-16.

GINET, R. - 1970 - Etude de la condenstation atmosphérique saisonnière dans la grotte de Hautecourt (Ain, France). C.R. Acad. Sc. Paris, 271, D, 1615-1618.

GINET, R. - 1971 - Biogéographie de Niphargus et Caecospheroma (Crustacés troglobies) dans les départements français du Jura et de l'Ain-Origine; influence des glaciations. Actes $4^{\circ}$ Cong. suisse Spéléol., Neuchâtel, sept. 70, 186-198.

GISLEN, T. and BRINCK, P. - 1950 - Subterranean waters on Gotland with special regard to the Lummelunda curren t. Lund Univ: s Arsskrift N.F. 2, 46, 6, 1-80.

HEIM DE BALSAC, H. - 1934 - Un Diptère peu connu de la faune française, Chionea lutescens luünds. (Dipt. Tipulidae). Précisions sur la biologie. Bull. Soc. ent. France, 39, $102-105$.

JEANNEL, R. - 194.3 - Les fossiles vivants des cavernes. Paris, Gallimard, 321 pp.

KRATOCHVIL, J. -- 1936 - Renseignements sur les espèces du genre Chionea de Tchécoslovaquie. Bull. Soc. ent. France, 41, 243-250.

MAGNIN, A. - 1886 - La végétation de la région lyonnaise et de la partie moyenne du bassin du Rhône. Bâle, Georg, 513 pp, 7 cartes.

MANI, M.S. - 1962 - Introduction to high altitude entomology. London, Methuen, $301 \mathrm{pp}$.

MANI, M.S. - 1968 - Ecology and biogeography of high altitude Insects. W. Junk N.V., La Haye. 527 pp.

PIERRE, C. - 1924 - Faune de France. 8. Diptères: Tipulidae. Paris, Lechevalier, 159 pp.

PEYERIMHOFF, P. de - 1906 - Recherches sur la faune cavernicole des Basses-Alpes. Ann. Soc. ent. France, LXXV, 203-211.

TURQUIN, M.J. - 1971 - Une biocoenose cavernicole originale pour le Bugey: le Puits de Rappe. 96 ${ }^{\circ}$ Cong. Soc. sav. Toulouse, sous-presse.

TURQUIN, M.J. - 1971 - La faune de la grotte de Hautecourt (Ain). Actes Cong. Chambéry A.F.A.S., sous-presse.

STRINATI, P. et AELLEN, V. - 1967 - Faune actuelle de deux grottes suisses de haute altitude. Rassegna Speologica Italiana, XIX, 1-2, 164.

VANDEL, A. - 1964 - Biospéologie. Paris, Gauthier-Villars, 619 pp. 